\title{
Magnetic Field Analysis of Plasma Guide in Galathea Trimyx
}

\author{
JIN Xianji , TONG Weiming, ZHOU Tianyi \\ Harbin Institute of Technology, CHINA
}

\begin{abstract}
You Galathea Trimyx is a kind of small size, multipole magnetic confinement devices in controlled thermonuclear fusion. Plasma guide is one of important part in Galathea Trimyx which is responsible for transporting fast and slow plasma bunches ejected from plasma gun. The distribution and uniformity of magnetic field in completed plasma guide is analyzed in detail, including in $x$-axis direction and in z-axis direction. On the basis, the motion of plasma in the guide is discussed.
\end{abstract}

\section{Introduction}

Plasma confinement by magnetic field is a very important issue for controlled thermonuclear fusion. Galathea Trimyx is a special setup ${ }^{[1]}$, whose difference from Tokamak is that the current-carrying conductor here is 'mixed' with the magnetic field ${ }^{[2]}$. In Galatheas, the plasma-immersed current-carrying conductors are called 'myxines'. In paper[3], the basic configuration and description of Galathea Trimyx is given. The block diagram of the Galathea Trimyx is shown in figure 1.

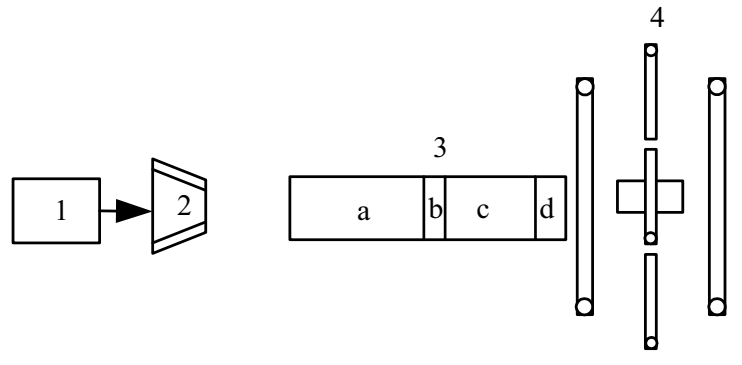

Fig. 1 Block diagram of Galathea Trimyx: (1) power supply, (2) plasma gun, (3) completed plasma guide, (4) Trimyx confinement system

In Galatea, the aim of plasma guide is to produce a quasi-uniform magnetic field whose length is about $1.2 \mathrm{~m}$. Generally, uniform magnetic field can be produced by a tightly wound solenoid, but this way is waste of materials. This configuration use coils which are amounted away a certain distance to produce uniform magnetic field ${ }^{[4]}$. In this paper, we first analyze the magnetic field distribution in the plasma guide, which is the basis of the plasma transport in the guide, and then analyze the transport in the guide from the point of view of the single particle motion $^{[5]}$.

\section{Magnetic field analysis of plasma guide}

The plasma guide are constituted by 19 identical thin coils $^{[6]}$ (110 $\mathrm{mm}$ in diameter), which can generate uniform magnetic field when a steady current follow though the coils at the same time. The whole guide model can be simplified by analyzing one single coil.

\subsection{Model of one single coil}

For simplicity, we analyze single coil first. In coordinate $O(x, y, z)$, the coil is placed on plane $x O y$. This coil is axisymmetric by $\mathrm{Oz}$ axis, the magnitude of $\boldsymbol{B}$ generated by this coil is also axisymmetric except that the direction of $\boldsymbol{B}$ beside the plane $x O y$ is different, so we only just need to analyze the $\boldsymbol{B}$ distribution in plane $x O z$.

Take an arbitrary point $P(x, 0, z)$ in plane $x O z$, which is shown in figure 2 .

By Biot-savart Law, we know the magnetic field ${ }^{[7]}$ generated by current infinitesimal $I \mathrm{~d} \boldsymbol{l}$ at point $P$ is:

$$
\mathrm{d} \boldsymbol{B}=\frac{\mu_{0}}{4 \pi} \cdot \frac{I \mathrm{~d} \boldsymbol{l} \times \boldsymbol{R}}{R^{3}}
$$

Where, $\mu_{0}$ is the permeability in vacuum, $I$ is the current.

The magnetic along $\mathrm{z}$-axis is:

$$
B_{z}=\frac{\mu_{0} I a^{2}}{2\left(a^{2}+z^{2}\right)^{\frac{3}{2}}}
$$

Let $z=k a$, equation(2) can be simplified:

$$
B_{z}=\left(1+k^{2}\right)^{-\frac{3}{2}} \frac{\mu_{0} I}{2 a}=f(k) \frac{\mu_{0} I}{2 a}
$$




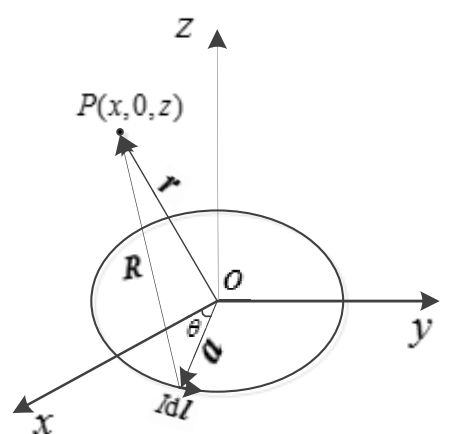

Fig. 2 Single coil analysis

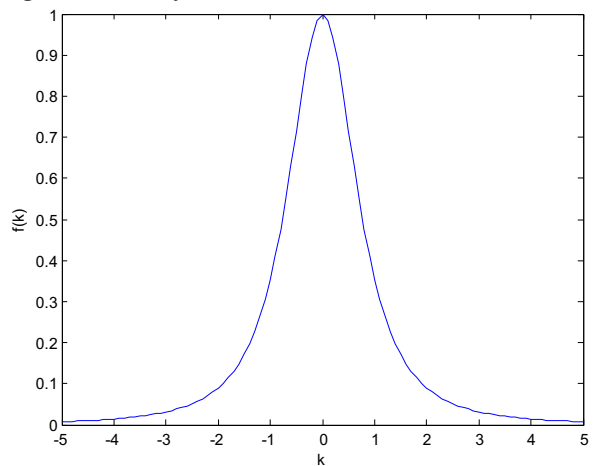

Fig. 3 Variation of $f(k)$

From figure 3 , when $\mathrm{k}=0, \mathrm{z}=0$, the magnitude of $\mathrm{B}$ is maximum; the $\mathrm{B}$ decreases $97 \%$ when $\mathrm{k}=3, \mathrm{z}=3 \mathrm{a}$. So we can take 7 series coils instead of the whole plasma guide, and this will not lead to too much error.

\subsection{Distribution of magnetic field in whole plasma guide}

The distribution of magnetic field in space produced by single coil can be calculated by equation(1), but the calculation process is very complicated ${ }^{[8]}$. The material of coils are copper, the coils are set multi-turn domain, the current flow through the coils is $200 \mathrm{~A}$, the space outside the coils is air.

Figure 4 shows the distribution of magnetic line in plasma guide. Figure 5 is the variation curve of $\mathrm{B}$ along the line which distance from the axis line is $10 \mathrm{~mm}$, $20 \mathrm{~mm}, 30 \mathrm{~mm}$, and $40 \mathrm{~mm}$. From figure 4 and figure 5, we can get conclusions: (1) from the $10 \mathrm{~mm}$ curve, the magnetic line in figure 4 is straight line, but the magnitude also has a small fluctuation; (2) all curves get its maximum at geometry center of coils, all curves have a same mean value $0.042 \mathrm{~T}$.

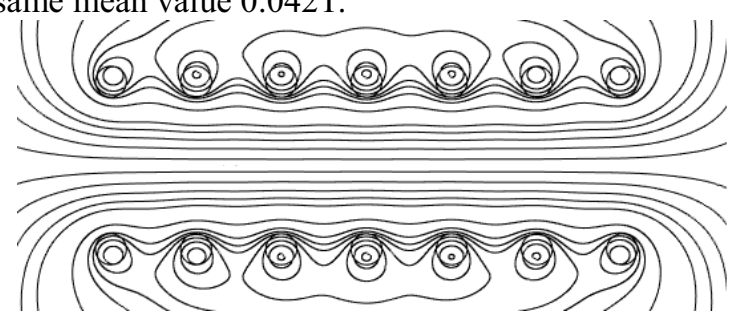

Fig. 4 Distribution of magnetic line in plasma guide

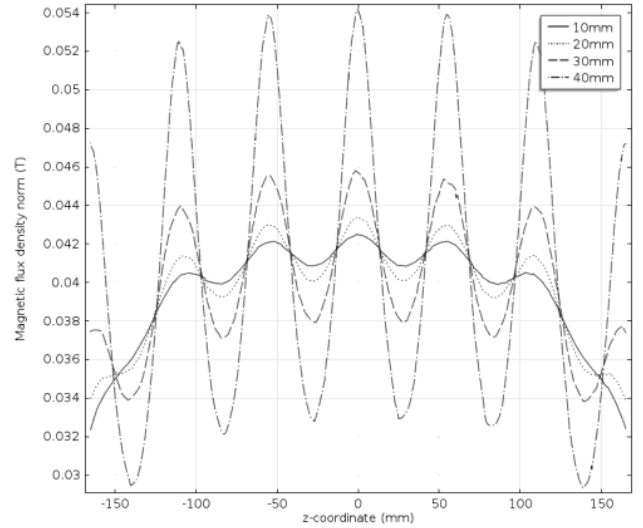

Fig. 5 Distribution of $\mathrm{B}$ along line at plasma guide aix

When $\mathrm{z}$ is a constant value, $\mathrm{B}$ can be considered as a dependence of $\mathrm{x}, B=B(x)$. Define the relative change rate of $\mathrm{B}$ as:

$$
\delta(x)=\frac{B(x)-B\left(x_{0}\right)}{B\left(x_{0}\right)} \times 100 \%
$$

Where $B\left(x_{0}\right) \longrightarrow$ magnitude of $B$ at axis line(T);

$B(x) \longrightarrow$ magnitude of $B$ at line $x \mathrm{~mm}$ away from axis line( $\mathrm{T})$;

On plane of coil 4 , get the B value at $x=0 \mathrm{~m}, 0.01 \mathrm{~m}$, $0.02 \mathrm{~m}, 0.03 \mathrm{~m}, 0.04 \mathrm{~m}$, calculate the relative change rate $\delta(x)$, the result is shown in Table 1 .

Tab. 1 The relative change rate in plasma guide

\begin{tabular}{|c|c|c|c|c|c|}
\hline$x(\mathrm{~m})$ & 0 & 0.01 & 0.02 & 0.03 & 0.04 \\
\hline$B(T)$ & 0.0339 & 0.0343 & 0.0354 & 0.0366 & 0.0421 \\
\hline$\delta$ & & $1.2 \%$ & $4.4 \%$ & $8.0 \%$ & $24.2 \%$ \\
\hline
\end{tabular}

From table 1, the magnetic field change rate is $8.0 \%$ at $x=0.03 \mathrm{~m}$, the rate increases to $24.2 \%$ at $x=0.04 \mathrm{~m}$. In this experiment, the required relative change rate is $10 \%$, so the magnetic field in the plasma guide can be separated into two kinds: cylindrical uniform magnetic field region which radius is $30 \mathrm{~mm}$ and magnetic mirror field region outside the cylindrical region.

\section{Plasma motion in plasma guide}

\subsection{Plasma motion in uniform magnetic field region}

By plasma diagnostic equipment, the main parameters of plasma beam from plasma gun to the entrance of plasma guide can be monitored. The parameters are as follows: the half-angle of the plasma jet divergence at the exit from the gun was $10^{\circ}$, the number of particles entering the plasma guide was about $10^{19}$, the average particle velocity of plasma bunch in the guide was $5 \times 10^{4} \mathrm{~m} / \mathrm{s}$.

By plasma physics, in uniform magnetic field, charged particle has a linear motion along B which is not affected by $\mathrm{B}$; in the plane perpendicular to $\mathrm{B}$, the charged particle has a cyclotron gyration motion. The cyclotron radius is $r_{\mathrm{L}}=\frac{m v_{\perp}}{|q| B}$, and the cyclotron 
frequency is $\omega_{\mathrm{c}}=\frac{|q| B}{m}$. Generally, compared to plasma radius, the cyclotron radius is very small, so particles can be constrained finely by magnetic field. We can classify the plasma into two kinds:

1. Plasma only have horizontal velocity when they get to the inlet of plasma guide, the velocity $v_{\|}=5 \times 10^{4} \mathrm{~m} / \mathrm{s}$, the plasma will have a uniform linear motion.

2. Plasma have a certain angle with horizontal direction when they get to the inlet of plasma guide, the maximum angle is $10^{\circ}$, horizontal velocity is $v_{\|}=4.92 \times 10^{4} \mathrm{~m} / \mathrm{s}$, vertical velocity is $v_{\perp}=0.868 \times 10^{4} \mathrm{~m} / \mathrm{s}$. Plasma will have a cyclotron motion, spiraling forward.

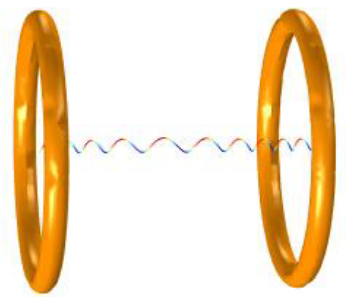

Fig. 6 Particle trajectory

\subsection{Plasma motion in uniform magnetic field area}

By plasma diagnostic device, some important parameters when plasma get through from plasma gun to the inlet of plasma guide can be monitored: the half-angle of the plasma jet divergence at the exit of gun is $10^{\circ}$, the number of particles entering the plasma guide is $10^{19}$, the average particle velocity in the plasma guide is $5 \times 10^{4} \mathrm{~m} / \mathrm{s}$, the injection duration being $30 \mu \mathrm{s}$, the energy of plasma bunch at the inlet is 20J. From the knowledge of plasma physics, in uniform magnetic, charged particles have a constant velocity along magnetic field lines and have a simple cyclotron gyration in the plane perpendicular to the magnetic line, the trajectory of a charged particle in space is a helix.

\subsection{Plasma motion in magnetic mirror field}

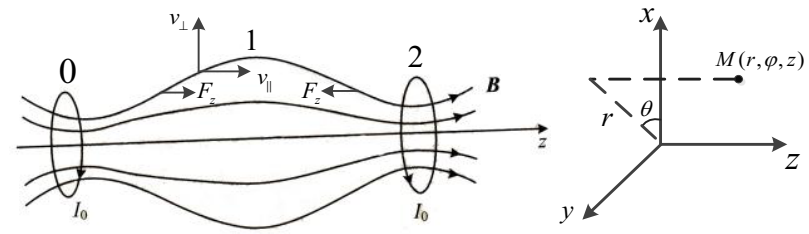

Fig. 7 Charged particle motion in magnetic mirror field

Figure 7 is simple magnetic mirror field, which is generated by two parallel current-carrying coil ${ }^{[9]}$. In cylindrical system, we have $B_{\varphi}=0, \frac{\partial}{\partial \varphi}=0$, $B_{r}=-\frac{1}{2} r_{L} \frac{\partial B_{z}}{\partial z}$. By the equation of motion ${ }^{[10]}$, the force on plasma along $z$ direction is:

$$
F_{z}=m \frac{\mathrm{d} v_{z}}{\mathrm{~d} t}=-q v_{\varphi} B_{r}=-\frac{1}{2} \frac{m v_{\perp}^{2}}{B} \frac{\partial B_{z}}{\partial z}
$$

Plasma will have a cyclotron motion whose orbit is a helix along magnetic line. From position 0 to 1 , charged particles are subjected to a horizontal force to the right because of the decrease of magnetic field $B_{z}$, the horizontal velocity $v_{\|}$increases, and the kinetic energy of particles is a constant, therefore the vertical velocity $v_{\perp}$ decreases; from position 1 to 2 , charged particles are subjected to a horizontal force to the left because of the increase of magnetic field $B_{\mathrm{z}}, v_{\|}$decreases, $v_{\perp}$ increases. If $v_{\|}$has not decrease to zero when it reaches position 2 , particle can get through this magnetic mirror field. The particle trajectory is shown in Figure 8 a); if the particle has not reach position 2 when $v_{\|}$decreases to zero, it will is subjected to a horizontal force to left again and accelerates to left, the particle trajectory is shown in Figure $8 \mathrm{~b}$ ). At the same time, the particles also have a guiding center shifting motion which direction along the velocity of cyclotron motion, plasma will not escape from the plasma guide.

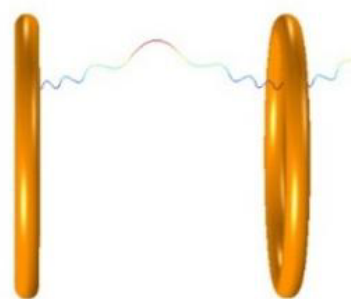

a) Particle passes through

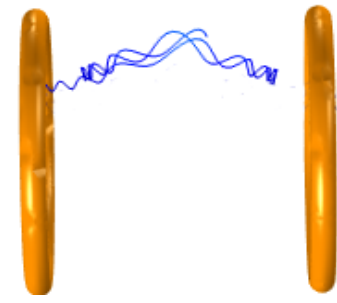

b) Particle reflects
Fig. 8 Particle trajectory in magnetic mirror field

\subsection{Plasma motion in plasma guide}

In software COMSOL Multiphysics, add Charged Particle Tracing Module to the plasma guide model, the two modules are coupled by magnetic flux density. The particles are set proton; the particles are released along $\mathrm{z}$ axis in the left-most coil plane; all the particles have same initial velocity; boundary conditions are set Freeze (when particles get this boundary, their positions and velocity are fixed). Simulating the plasma guide under these two modules, the plasma motion trajectory is shown in figure 9.

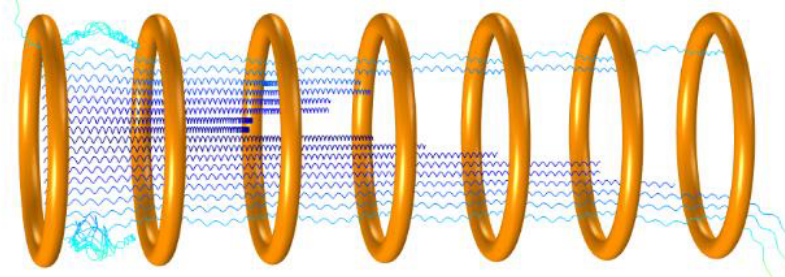

Fig. 9 Particle trajectory in whole plasma guide

From the figure, most particles can pass through the plasma guide, only a little fraction of particles escape from guide, and this phenomenon happens in the first magnetic mirror field. Whether particles escape from the plasma guide is decided by the position and velocity they 
enter in the guide. Under the same injection direction and same motion time, particle in the side of coil run further than that in the center of coil, this because the B get maximum at the center of plasma guide, according to $T=m v / q B$, plasma cyclotron gyration period getting shorter.

\section{Impact of plasma gun jet angle to particle motion}

According to the discussion in the last chapter, whether particles escape from the plasma guide is decided by the position and velocity they enter in the guide, the particle initial velocity depend on plasma gun jet angle. In current Galatea configuration, the half-angle of the plasma jet divergence at the exit of gun is $10^{\circ}$. While maintaining the structure parameters of plasma guide unchanged, we analyze the impact of plasma gun jet angle to particle motion.

\subsection{Experiment design}

No matter what the $v_{\|}$and $v_{\perp}$ is, the particle in the uniform magnetic field has a cyclotron motion, and the cyclotron gyration motion radius is very small compared to the guide radius, so the plasma gun jet angle has no effect to the particle in the uniform magnetic field, just need to analyze the particle motion in the magnetic mirror field. Assume $r$ is the distance between the point in the coil plane and the axis, set 4 group different plasma gun jet angle, respectively, $20^{\circ}, 40^{\circ}, 60^{\circ}, 80^{\circ}$, the halfangle is $10^{\circ}, 20^{\circ}, 30^{\circ}, 40^{\circ}$. The following analysis is taken by half-angle because the plasma beam ejected from gun is axisymmetric. The values of $v_{\|}$and $v_{\perp}$ are shown in table 2 .

Tab. 2 Velocity distribution in different plasma gun jet angle

\begin{tabular}{|c|c|c|c|c|}
\hline Number & $\begin{array}{c}\text { Half- } \\
\text { angle(deg) }\end{array}$ & $\begin{array}{c}\text { Plasma } \\
\text { velocity }(\mathrm{m} / \mathrm{s})\end{array}$ & $v_{\|}(\mathrm{m} / \mathrm{s})$ & $v_{\perp}(\mathrm{m} / \mathrm{s})$ \\
\hline 1 & 10 & $5 \times 10^{4}$ & $4.92 \times 10^{4}$ & $0.868 \times 10^{4}$ \\
\hline 2 & 20 & $5 \times 10^{4}$ & $4.7 \times 10^{4}$ & $1.71 \times 10^{4}$ \\
\hline 3 & 30 & $5 \times 10^{4}$ & $4.33 \times 10^{4}$ & $2.5 \times 10^{4}$ \\
\hline 4 & 40 & $5 \times 10^{4}$ & $3.83 \times 10^{4}$ & $3.21 \times 10^{4}$ \\
\hline
\end{tabular}

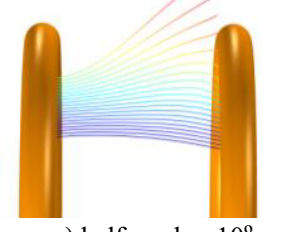

a) half-angle $=10^{\circ}$

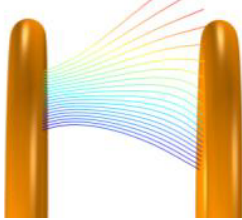

c) half-angle $=30^{\circ}$

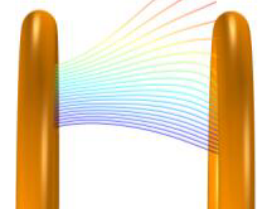

b) half-angle $=20^{\circ}$

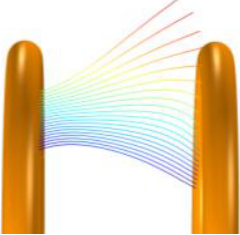

d) half-angle $=40^{\circ}$
Fig. 10 Plasma trajectory under different half-angle

4.2 Analysis of simulation result
The particle trajectory simulation is show in Fig. 1. All the four sub-figure has 5 particles free from the constraint of magnetic mirror field and escape out the plasma guide. These 5 particles all released at position $r=(45,50)$, but they have different $v_{\|}$and $v_{\perp}$. This experiment demonstrates that whether the particles escape from the guide is decided by the initial position when they enter the first magnetic mirror field, and is irrelevant with the plasma gun jet angle. When we taking plasma magnetic confinement experiment in the laboratory, the installation placement of plasma gun should make sure plasma beam within the range of $45 \mathrm{~mm}$ as they get to the entrance of plasma gun.

\section{Conclusions}

By theoretical and mathematical analysis of magnetic field in plasma guide, we can get some conclusions:

1. The whole plasma guide can be simplified as 7 coils by analyzing the magnetic field distribution of single coil.

2. In analysis of common plasma guide, the magnetic relative change rate $\delta<10 \%$ when $x<30 \mathrm{~mm}$, and the magnitude is close to $0.034 \mathrm{~T}$, which corresponds well to the data monitored from experiment.

3. The installation placement of plasma gun should make sure plasma beam within the range of $45 \mathrm{~mm}$ as they get to the entrance of plasma gun.

\section{References}

1. A.I.Morozov,A.I.Bugrova, et al (2006) Effect of Magnetic Barrier on the Plasma Parameters in a Trimyx-M Galatea. Technical Physics Letters. vol. 32, no.11, pp.939-941

2. Kenro Miyamoto(1981) Controlled fusionand plasma physics, JIN Shangxian, translated, CA:Beijing, China, pp.533-536

3. A.I.Morozova, A.I.Bugrovab, et al(2007) Plasma Parameters in the graded Trimyx-M Galathea. Technical Physics, vol.52, no. 12 , pp.1546-1551

4. Kaganovich, I.D. Startsev, et al(2007) Charge and current neutralization of an ion-beam pulse propagating in a background plasma along a solenoidal magnetic field. Physical Review Letters, vol.99, no.23, pp.235002

5. Khanh T. Nguyen, John A. Pasour et al(2009) Intense Sheet Electron Beam Transport in a Uniform Solenoidal Magnetic Field. IEEE Transactions on Electron Devices, vol.56, no.5, pp.744-752

6. A. I.Morozov,A .I. Bugrova, at al(2006) Injectionof Plasmainto the Trimyx Galathea, vol. 32, no.3, pp.195-206

7. Xianwei Wang, Fei Xie, et al(2014) Electromagnetic Analysis of the ITER Upper VS Coil. J Supercond Nov Magn. vol.27, no.1, pp.1015-1019

8. Babic, S.I. Akyel(2006) New mutual inductance calculation of the magnetically coupled coils: Thin disk coil-thin wall solenoid. Journal of Electromagnetic Waves and Applications, vol. 20,no.10, pp.1281-1290

9. Liu Hui, Chen Peng-Bo, et al(2015) Particle-in-cell simulation for different magnetic mirror effects on the plasma distribution in a cusped field thruster. Chinese Physics B, vol. 24, no.8, pp.085-202 